\title{
Noise, Information, and the Favorite-Longshot Bias in Parimutuel Predictions*
}

\author{
Marco Ottaviani $^{\dagger} \quad$ Peter Norman Sørensen $^{\ddagger}$
}

January 2009

\begin{abstract}
According to the favorite-longshot bias, the expected return on an outcome tends to increase in the fraction of bets laid on that outcome. We derive testable implications for the direction and extent of the bias depending on the ratio of private information to noise present in the market. We link this ratio to observables such as the number of bettors, the number of outcomes, the amount of private information, the level of participation generated by recreational interest in the event, the divisibility of bets, the presence of ex post noise, as well as ex ante asymmetries across outcomes.
\end{abstract}

Keywords: Parimutuel betting, favorite-longshot bias, private information, noise, lotteries.

JEL Classification: D82 (Asymmetric and Private Information), D83 (Search; Learning; Information and Knowledge), D84 (Expectations; Speculations).

${ }^{*}$ We thank the referees, Albert Banal-Estañol, Jimmy Chan, Jim Dana, Renato Gomes, Marianne Hinds, Chris Jones, Frédéric Koessler, Gustavo Manso, Stefano Sacchetto, Russell Sobel, Richard Zeckhauser, Bill Ziemba, and seminar participants at Bocconi, Cambridge, Cornell, Duke, INSEAD, Northwestern, Paris, Oxford, Texas A\&M, the 4th International Equine Industry Program Academic Conference in Louisville, the 2005 CEPR European Summer Symposium in Economic Theory at Gerzensee, the 2005 International Conference on Finance at Copenhagen, the 2005 World Congress and the 2006 North American Winter Meetings of the Econometric Society for helpful comments.

${ }^{\dagger}$ London Business School and Kellogg School of Management, Northwestern University, 2001 Sheridan Road, Evanston, IL 60208-2013. Phone: +1-847-467-0684. Fax: +1-843-467-1777. E-mail: m-ottaviani@northwestern.edu. Web: http://faculty.london.edu/mottaviani.

¥Department of Economics, University of Copenhagen, Studiestræde 6, DK-1455 Copenhagen K, Denmark. Phone: +45-3532-3056. Fax: +45-3532-3000. E-mail: peter.sorensen@econ.ku.dk. Web: http://www.econ.ku.dk/sorensen. 
Betting and prediction markets provide a natural environment for testing theories of decision making under uncertainty and price formation. The uncertainty about the value of the assets traded in these markets is resolved unambiguously and the outcome is observed publicly. In most cases it is also reasonable to presume that the realized outcomes are exogenous with respect to market prices. In regular financial markets, in contrast, the intrinsic value of assets is observed only in the long run (if ever) and is often affected by prices.

In racetrack betting and lottery games throughout the world, a widely adopted market structure is parimutuel wagering. ${ }^{1}$ According to the parimutuel system, the holders of winning tickets share the total amount of money bet on all outcomes in proportion to their bets, net of the takeout for taxes and expenses. The parimutuel odds that are paid out to winning bets (as a multiple of the amount wagered) depend on the overall distribution of bets and are thus determined by the wagering public itself. Because these odds are not skewed by price-setting suppliers (such as bookmakers), parimutuel betting markets are particularly well suited to testing market efficiency. ${ }^{2}$

The most widely documented empirical regularity observed in horse-betting markets is the favorite-longshot bias (hereafter, FLB): horses with "short" odds (i.e., favorites) tend to win more frequently than indicated by their odds, while horses with "long" odds (i.e., longshots) win less frequently. ${ }^{3}$ Consequently, the expected returns on longshots are lower than on favorites. This finding is puzzling, because expected returns are not equalized across horses. To further add to the puzzle, note that for parimutuel lottery games, such as Lotto, a reverse FLB always results: the expected payoff is lower on numbers that attract a higher-than-proportional fraction of bets. ${ }^{4}$

\footnotetext{
${ }^{1}$ All horse-race betting in the U.S. is parimutuel. This mutual system is also used in greyhound racing, jai alai, and other sporting events in which participants finish in a ranked order. Recently, the parimutuel structure has been adopted in a number of prediction markets, where claims contingent on various economic indices are traded. As Nicholas Economides and Jeffrey Lange (2005) document, these markets allow traders to hedge risks related to the release of U.S. nonfarm payroll employment data and European harmonized indices of consumer prices.

${ }^{2}$ In parimutuel markets, the demand for bets on one outcome generates the supply on all other outcomes. See Steven D. Levitt (2004) for a discussion of supply-driven distortions in betting markets not using the parimutuel system.

${ }^{3}$ For surveys of the extensive literature, we refer to Richard H. Thaler and William T. Ziemba (1988), Donald B. Hausch and Ziemba (1995), Raymond D. Sauer (1998), Bruno Jullien and Bernard Salanié (2008), and Ottaviani and Sørensen (2008).

${ }^{4}$ See David Forrest, O. David Gulley, and Robert Simmons (2000) for a test of rational expectations in lotteries. The reverse FLB is also present in some betting markets (see e.g. Kelly Busche and Christopher D. Hall 1988, Leighton Vaughan Williams and David Paton 1998, and our discussion below).
} 
This paper develops a theory that can explain the variation in the occurrence of the FLB and its reverse across parimutuel betting markets. In the model, bettors decide simultaneously whether, and on which of several outcomes, to bet. Each bettor's payoff has two components. First, there is a "common value" component, equal to a bet's expected monetary payoff according to the final odds. ${ }^{5}$ Second, bettors derive a private utility from gambling. ${ }^{6}$ For simplicity, we set this "recreational value" to be the same for all bettors and outcomes. ${ }^{7}$

To characterize the effect of private information on market outcomes, we allow bettors to have heterogeneous beliefs based on a common prior and the observation of private signals. While private information is clearly absent in lottery games, there is widespread evidence of its presence in horse betting. ${ }^{8}$ In a limit case relevant for lottery games, the private signals contain no information.

We model betting as a simultaneous move game. This is a realistic description of lottery games, in which the numbers picked by participants are not made public before the draw. For betting on horse races, the distribution of bets (or, equivalently, the provisional odds given the cumulative bets placed) is typically displayed over time on the tote board and updated at regular intervals until post time, when no more bets are accepted. However, a large proportion of bets are laid in the very last seconds before post time. ${ }^{9}$ Thus, we focus on the last-minute simultaneous betting game and characterize the symmetric Bayes-Nash equilibrium. ${ }^{10}$

Building on the equilibrium structure, we characterize the surprise generated by the ex post realization of the actual bets. The sign and extent of FLB depend on the amount of information relative to noise that is present in the realized bets. We show that if the population of informed bettors is large and/or private information is sufficiently precise, then ex post favorites are more likely to win than the realized market odds indicate at face value - and the opposite is true for longshots. If an outcome turns out to attract a larger

\footnotetext{
${ }^{5}$ By assuming risk neutrality, we depart from a large part of the betting literature since Martin Weitzman (1965) in which bettors are risk loving. Section 6 discusses this and other alternative theories.

${ }^{6}$ See, for example, John Conlisk (1993).

${ }^{7}$ In the case of parimutuel derivative markets mentioned in footnote 1 , the private value derives from the benefit of hedging against pre-existing risks correlated with the outcome on which betting takes place.

${ }^{8}$ See Section 6.

${ }^{9}$ See National Thoroughbred Racing Association (2004).

${ }^{10}$ Ottaviani and Sørensen (2006) endogenize the timing of bets in a dynamic model. They show that small privately informed bettors have an incentive to wait until post time and thus end up betting simultaneously.
} 
fraction of bets (and the associated odds are shorter), more bettors must have privately believed that this outcome was likely. Hence, the occurrence of short odds indicates favorable information. When this information is sufficiently strong, the FLB results, as observed in horse betting.

When bettors are less informed, the realized bets contain less information and are more affected by noise. To understand the effect of noise, apply our methodology to Lotto. Bettors' signals are completely uninformative about the outcome. Market odds will again vary randomly, depending on the noisy realization of the bets. Given that all numbers are equally likely to be drawn, by construction the expected payoff is lower for those numbers that attract more than their fair share of bets. This is because the jackpot is shared among the lucky few who pick the winning number, according to the parimutuel rule. Thus, lottery outcomes with short market odds yield automatically lower expected returns than outcomes with long market odds. More generally, when signals contain little information, and there is aggregate uncertainty about the final distribution of bets due to noise, our model predicts a reverse FLB - as is observed in parimutuel lotteries.

The paper proceeds as follows. Section 1 casts our contribution within the theory literature. Section 2 formulates our general model with an arbitrary number of outcomes. Section 3 characterizes the different equilibrium regimes, depending on the level of participation in the market. Focusing on the case in which all outcomes are ex ante equally likely and the recreational value is so large that no bettor abstains, Section 4 develops our ex post comparison between market and posterior probabilities, based on the surprise effect. This symmetric model is ideal for uncovering the role of noise and information in generating the FLB or its reverse. Section 5 examines the comparative statics properties of the FLB by analyzing the effect of exogenous changes in parameters on the ratio of private information to noise present in equilibrium. Mostly within tractable special cases, we illustrate how the extent of the FLB varies with respect to the amount of private information, the number of bettors, the number of outcomes, the divisibility of bets, the presence of ex post noise, factors (such as the recreational interest in the event or the takeout rate) that affect the bettors' participation decision, and ex ante asymmetries across outcomes. Section 6 compares our explanation of the empirical evidence with the main alternatives proposed in the literature. Section 7 concludes. Appendix A collects the proofs of all propositions. Appendix B reports details on a tractable example using the Dirichlet distribution, including 
the proofs of all lemmas.

\section{Literature}

This paper builds on the informational explanation for the FLB proposed by Ottaviani and Sørensen (forthcoming) in a simple model without noise. ${ }^{11}$ To develop testable implications for the extent of the FLB across different environments, this paper analyzes a general version of the model which allows for noise and endogenizes the level of market participation. We argue that the comparative statics predictions of the model are broadly in line with evidence from parimutuel games characterized by different levels of information relative to noise.

The informational explanation investigated here is fundamentally different from Mukhtar M. Ali's (1977) Theorem 2, which derives the FLB from heterogeneity of bettors' prior beliefs. ${ }^{12}$ We assume instead that bettors share a common prior belief, but possess private information. In addition, while Ali's explanation hinges on ex ante asymmetries in the probabilities of the different outcomes, our explanation is valid also when the outcomes are all ex ante equally likely. To isolate our informational mechanism, our baseline analysis focuses on a fully-symmetric environment.

Hyun Song Shin (1991 and 1992) formulates an information-based explanation of the FLB in the context of fixed-odds markets, in which prices are determined by bookmakers. Even though our informational assumptions are similar to Shin's, the logic and the extent of the FLB are different in the two markets. While Shin's explanation for the bias relies on ex ante asymmetries, our explanation in the context of parimutuel markets does not. ${ }^{13}$

The first game-theoretic analysis of parimutuel markets with private information is by Frédéric Koessler, Charles Noussair, and Anthony Ziegelmeyer (2008). They construct

\footnotetext{
${ }^{11}$ Ottaviani and Sørensen's (forthcoming) is essentially the limit version of the model of this paper as the number of bettors per outcome goes to infinity (so that noise disappears by the law of large numbers) and bettors are forced to participate.

${ }^{12}$ See also Émile Borel (1938), Edmund Eisenberg and David Gale (1959), Sumir Chadha and Richard E. Quandt (1996), Lawrence D. Brown and Yi Lin (2003), and Justin Wolfers and Eric Zitzewitz (2004) for analyses of prediction markets with heterogeneous prior beliefs across bettors, in the absence of private information.

${ }^{13}$ In addition, Ottaviani and Sørensen (2005b) show that the parimutuel payoff structure involves a builtin insurance against the winner's curse. In parimutuel markets, an increase in the number of informed bettors tends to make market odds more extreme, thereby reducing the FLB. In fixed-odds markets, in contrast, an increase in the fraction of informed bettors strengthens the FLB, because adverse selection is worsened.
} 
equilibria when bettors have binary signals. Our analysis is simplified by assuming instead that bettors have continuously distributed signals, as in other auction-theoretic models of price formation. We are then able to characterize and quantify the FLB, endogenize the bettors' participation decision, and derive a number of empirical predictions.

\section{Model}

Bets can be placed on the realization of a random variable, $k \in\{1, \ldots, K\}$, where $K \geq$ 2 is the number of possible outcomes. In Lotto games, $k$ corresponds to the winning combination. For bets on the win pool in a horse race, $k$ represents the identity of the winning horse. The model applies more generally to pools on outcome combinations within and across races. ${ }^{14}$

There are $N$ bettors with a common prior belief distribution $q$, where $q_{k} \geq 0$ is the prior probability of outcome $k .{ }^{15}$ Bettor $i \in\{1, \ldots, N\}$ is privately endowed with signal $s^{i}$, leading to the private (posterior) belief distribution $p^{i}$. Bettors differ only because of the realization of their private signals, but they are identical ex ante: the joint distribution of private beliefs and the state is unchanged if the identities of the players are switched. ${ }^{16}$ We assume that this joint distribution of private beliefs is continuous. For any individual, the density of the private belief conditional on outcome $k$ is denoted by $g(p \mid k)$.

On the basis of the private belief, each bettor decides the outcome on which to bet a fixed and indivisible amount, normalized to 1 , or to abstain from betting. ${ }^{17}$ All bettors are risk neutral and maximize the expected monetary payoff, plus a fixed recreational utility value received from betting. This recreational value $(u \geq 0)$ is foregone when a bettor abstains, and hence generates a demand for betting. ${ }^{18}$

\footnotetext{
${ }^{14}$ For example, a race with $L$ horses thus has $K=L(L-1)$ "exacta" outcomes, consisting in the winner and the runner-up in a given race. Some exotic bets have a very large number of outcomes. In the "pick six" pool, for instance, bettors are asked to guess the winners in six consecutive races, usually the second through the seventh in a given day. If there are $L$ horses in each race, the pick six pool admits $K=L^{6}$ combinations.

${ }^{15}$ We assume common prior to isolate the effect of asymmetric information. See Ottaviani and Sørensen (2005a) on the interaction of heterogeneous prior beliefs and private information.

${ }^{16}$ The assumption that bettors are ex ante identical is not essential for our results, but allows us to obtain a closed-form solution for the equilibrium. See Ottaviani and Sørensen (2006) for a model in which instead some bettors are outsiders (motivated by recreation) while other bettors are privately-informed insiders.

${ }^{17}$ See Rufus Isaacs (1953) and Ottaviani and Sørensen (2006) for analyses in which bettors can choose how much to bet. See Section 5.3 for a discussion of the effect of allowing bets to be divisible.

${ }^{18}$ Without this recreational utility, there is no betting in equilibrium, as predicted by the no-trade
} 
The total amount of money bet on all $K$ outcomes is placed in a common pool, from which a takeout fraction, $\tau \in[0,1)$, is subtracted for taxes and other expenses incurred to run the game. The remaining money is returned to those who bet on the winning outcome, $k$. We assume that there is no payment to the bettors when no bets were placed on the winning outcome. ${ }^{19}$ Let $b_{k}$ denote the total amount bet on $k$. If $k$ is the winning outcome, then every unit bet on $k$ receives the monetary payoff $(1-\tau)\left(\sum_{l=1}^{K} b_{l}\right) / b_{k}$. The market probability of outcome $k$ is equal to the fraction of money bet on that outcome, $b_{k} /\left(\sum_{l=1}^{K} b_{l}\right)$.

To ease the exposition, we present our formal results in terms of market probabilities, but it is easy to derive equivalent implications for odds. In betting markets, the odds on outcome $k$ are typically defined as the amount of money paid by the system for each dollar laid on that outcome, in addition to the dollar wagered. In order to balance the budget for any outcome realization, the parimutuel odds are $(1-\tau)\left(\sum_{l=1}^{K} b_{l}\right) / b_{k}-1$. Given the one-to-one correspondence between market odds and probabilities, our results can be immediately rephrased in terms of odds.

The strategy of a bettor maps every private belief into one of the $K+1$ actions: bet on outcome $k$ or abstain. In equilibrium, every bettor correctly conjectures the strategies used by the opponents and then plays the best response to this conjecture. By assumption, the game is always symmetric with respect to the players. Throughout the paper, we focus on symmetric equilibria; that is, on Bayes-Nash equilibria in which all bettors use the same strategy, mapping private beliefs into actions. ${ }^{20}$

\section{Equilibrium Regimes}

We begin by computing the expected payoff of a bettor. A bettor's payoff conditional on winning is random, because of the uncertainty present in opponent signals and bets. By risk neutrality, a bettor who assigns probability $p_{k}$ to outcome $k$ obtains an expected payoff equal to $p_{k} W_{k}-1+u$ when betting on outcome $k$, where $W_{k}$ is the expected payment from the pool to a bet on $k$, conditional on the realization of outcome $k$. Given the opponents'

\footnotetext{
theorem (Paul Milgrom and Nancy Stokey 1982).

${ }^{19}$ Our results continue to hold qualitatively with alternative rules on how the pool is split when no one bets on the winner. For example, the pool could be divided equally among all active bettors.

${ }^{20}$ Koessler, Noussair, and Ziegelmeyer (2008) note that there also may be asymmetric equilibria when $N$ is small.
} 
strategies, each bettor can calculate $\eta(l \mid k)$, the probability that an opponent bets on outcome $l$ when $k$ is the winning outcome. Using elementary probability theory, we can explicitly compute the expected payment, $W_{k}$, as a function of the betting probabilities $\eta(l \mid k)$ :

Proposition 1 Suppose that $N-1$ opponents bet on the outcomes $1, \ldots, K$ with conditional probabilities $\eta(1 \mid k), \ldots, \eta(K \mid k)$. Then the expected payment from the pool to a bet on $k$ conditional on the realization of outcome $k$ satisfies

$$
W_{k}= \begin{cases}(1-\tau) \frac{\sum_{l} \eta(l \mid k)-\left[\sum_{l \neq k} \eta(l \mid k)\right][1-\eta(k \mid k)]^{N-1}}{\eta(k \mid k)} & \text { if } \eta(k \mid k)>0 \\ (1-\tau)\left[1+(N-1) \sum_{l \neq k} \eta(l \mid k)\right] & \text { if } \eta(k \mid k)=0 .\end{cases}
$$

We are now ready to characterize the equilibrium of the game. There are three equilibrium regimes, depending on the level of recreational value $u:^{21}$

Proposition 2 Assume that the distribution of private beliefs is continuous with full support. There exists a uniquely defined critical value, $u^{*(N)} \in(\tau, 1)$, such that:

1. if $u \geq u^{*(N)}$, there exists a symmetric equilibrium in which all bettors bet actively,

2. if $u \in\left(\tau, u^{*(N)}\right)$, in any symmetric equilibrium some (but not all) of the bettors abstain, and such an equilibrium exists,

3. if $u \leq \tau$, all bettors abstain in the only symmetric equilibrium.

When the recreational value is sufficiently high or, equivalently, the takeout rate is sufficiently low, even bettors without strong private beliefs bet on one of the horsesthis is the "no abstention" regime on which we focus for most of the paper. With low recreational value or high takeout rate, bettors without strong private beliefs prefer not to place any bet - this is the "partial abstention" regime that we analyze in Section 5.5. Additional increases in the takeout rate further reduce participation, until the market completely breaks down by the logic of the no-trade theorem. ${ }^{22}$

\footnotetext{
${ }^{21}$ Proposition 2 can be extended to the case in which support is not full. Let $\bar{p}$ denote the maximal belief that any bettor attaches to any outcome. Let $\bar{u}=1-\bar{p}(1-\tau)$. Then $u^{*}>\bar{u} \geq \tau$ and cases 1 and 3 of Proposition 2 hold as stated. The claim in case 2 holds when $u \in\left(\bar{u}, u^{*}\right)$. If $u \in(\tau, \bar{u}$, our proof implies a weaker version of case 3 , that there exists an equilibrium in which every bettor abstains.

${ }^{22}$ Bettors with beliefs $p=1$ and $p=0$ always participate, but these beliefs have probability zero by the assumption that the belief distribution is atomless.
} 


\section{Market vs. Posterior Probabilities}

This section develops our theory of the FLB as an ex post surprise effect. Given that bettors are privately informed, and that their behavior depends on their private beliefs, the realized market probabilities contain information about the chance of different outcomes. In our Bayes-Nash equilibrium - as in parimutuel betting markets on horse race outcomes - bettors are surprised when they see the realization of the market probability, but they are unable to adjust the positions they have already taken. ${ }^{23}$

Applying Bayes' rule, for each market probability we can compute the corresponding posterior probability belief held by an outside observer (with a prior belief equal to the common prior). The idea is to compare each realized market probability with the corresponding posterior probability that incorporates the information revealed to the outside observer. As we explain below, the empirical probability can be identified with the posterior probability. The thrust of our approach consists of examining factors that affect the systematic relation between market and posterior - and thus empirical - probabilities.

To present our results in the clearest way, for most of the paper (up to Section 5.6) we focus on the symmetric case in which all outcomes are ex ante equally likely $\left(q_{k}=1 / K\right)$ and the posterior beliefs are symmetrically distributed. Thus, the belief distribution remains unchanged following a permutation of the identities of the $K$ outcomes. In addition, we posit that signals are conditionally independent and identically distributed across bettors. Up to Section 5.5, we further prevent any abstention by assuming that $u$ is sufficiently large.

The symmetric case is also an important empirical benchmark. Horse races are typically designed to be balanced in order to assure that their outcome is genuinely uncertain. Horses are sorted into categories depending on their observable characteristics, and known differences are eliminated in part by burdening the advantaged horses with additional weights. While asymmetries are never perfectly eliminated, these procedures are intended to reduce the presence of strong ex ante favorites or ex ante longshots.

Proposition 3 If $u$ is sufficiently large and signals are symmetrically distributed, it is a

\footnotetext{
${ }^{23}$ Any bias would be eliminated if bettors could instead adjust their positions after observing the realized market probabilities, as in a rational expectations equilibrium. However, the information on the final odds is typically not available to bettors because a substantial amount of bets are placed at the end of the betting period when the final odds are not yet determined, as in our simultaneous-move game.
} 
symmetric equilibrium to bet on the most likely outcome, that is, on the $k$ which maximizes $p_{k}$. If bettors have private information, then the probability that any bettor bets on the winner, $\eta(k \mid k)$, exceeds $\eta(l \mid k)=[1-\eta(k \mid k)] /(K-1)$, the probability that any bettor bets on any other outcome $l \neq k$.

Empirical investigations of the efficient market hypothesis proceed by grouping observations into classes according to their market probabilities, computed from final market odds. When $n$ bets are placed on outcome $k$, the implied market probability for outcome $k$ is $\pi_{k}=n / N$, equal to the fraction of money bet on this outcome. Empiricists then compute the empirical fraction of races that are won by horses belonging to each observation class. As first noticed by Richard M. Griffith (1949), the comparison of market with empirical probabilities typically reveals systematic differences between these probabilities. $^{24}$ At one end of the spectrum, large market probabilities (associated to favorites) tend to be smaller than the corresponding empirical probabilities. At the other end of the spectrum, longshots tend to win less frequently than indicated by their (already low) market probabilities.

We are now ready for the key step in our analysis. Based on the realized market probability for outcome $k$, an outside observer can update the prior to the posterior probability for this outcome, denoted by $\beta_{k}$. Because signals are random, bets on a given outcome follow a binomial distribution. When exactly $n$ bets are placed on $k$, Bayes' rule then yields

$$
\beta_{k}=\frac{q_{k} \operatorname{Pr}(\text { bets } \mid k \text { true })}{\operatorname{Pr}(\text { bets })}=\frac{\eta(k \mid k)^{n}[1-\eta(k \mid k)]^{N-n}}{\eta(k \mid k)^{n}[1-\eta(k \mid k)]^{N-n}+(K-1) \eta(k \mid l)^{n}[1-\eta(k \mid l)]^{N-n}},
$$

where $l \neq k$. The law of large numbers guarantees that the empirical frequency of outcome $k$ across many repetitions of the game is approximately equal to this posterior probability, $\beta_{k}$. By incorporating the information revealed in the betting distribution and adjusting for noise, this posterior probability is the correct estimate of the empirical probability of outcome $k$.

In our model, the systematic relation between posterior and market probabilities depends on the interplay between the amount of noise and information contained in the bettors' signals. To appreciate the role played by noise, note that even with very few bettors, market probabilities can range from zero to one. For example, if most bettors happen

\footnotetext{
${ }^{24}$ See footnote 3 for references to surveys of the empirical literature.
} 
to draw signals that lead them to believe $k$ is the most likely outcome, then the market probability for outcome $k$ would be very high. However, when the signals contain little information, the posterior probability is close to the prior. In this case, deviations of the market probability from the prior should be largely attributed to randomness contained in the signal. Then the reverse FLB is present: market probabilities are more extreme than posterior probabilities.

To illustrate the role of information relative to noise, consider a setting with $K=2$ outcomes and set the probability of a correct bet at $\eta(k \mid k)=2 / 3$. Suppose that the number of bettors $N$ is odd and focus on the betting realizations in which exactly one more bet is placed on outcome 1 than on outcome 2 . The informational content of the first $(N-1) / 2$ bets placed on either outcome is identical and cancels out. However, the chance of the last bet is twice as large when 1 is the winner than when 2 is the winner. Hence, the posterior probability of 1 being the winner is $2 / 3$. On the other hand, the market probability for outcome 1 is $(N+1) /(2 N)$. This number is strictly decreasing in $N$ and equal to the posterior probability $2 / 3$ when $N=3$. Noise dominates information when the number of bettors is small $(N=1)$, resulting in a reverse FLB. As the number of bettors increases, realized market bets contain more and more information, so that the posterior associated to any given market probability becomes more extreme. This implies the FLB, which results here with $N>3$ bettors. More generally, we have:

Proposition 4 Assume that the belief distribution is symmetric and that $u$ is so large that no bettor abstains. Let $\pi^{*} \in(0,1)$ be defined by

$$
\pi^{*}=\frac{\log \left(\frac{1-\eta(k \mid l)}{1-\eta(k \mid k)}\right)}{\log \left(\frac{1-\eta(k \mid l)}{1-\eta(k \mid k)}\right)+\log \left(\frac{\eta(k \mid k)}{\eta(k \mid l)}\right)}
$$

for any pair $l \neq k$. Take as given any market probability $\pi_{k} \in(0,1)$ for outcome $k$. As the number of bettors, $N$, becomes sufficiently large, a longshot's market probability $\pi_{k}<\pi^{*}$ (respectively, a favorite's $\pi_{k}>\pi^{*}$ ) is strictly greater (respectively, smaller) than its associated posterior probability, $\beta_{k}$.

By definition (3), $\pi^{*}$ is a proportion of bets on outcome $k$ that is neutral, i.e., at which the posterior belief is precisely equal to the prior. 


\section{Testable Implications}

We now derive testable implications of our theory by investigating a number of comparative statics properties that follow from Proposition 4. Exogenous changes in the environment affect the amount of information relative to noise contained in the bets, thereby determining the sign and extent of the FLB. Until Section 5.6, we maintain the assumption that the game is ex ante symmetric across outcomes.

\subsection{Information}

How does the FLB depend on the amount of information revealed in equilibrium? To answer this question for the symmetric case with $K=2$ outcomes, we define the informativeness of bets as the likelihood ratio $\eta(1 \mid 1) / \eta(1 \mid 2)=\eta(2 \mid 2) / \eta(2 \mid 1)$, which determines the extent of updating following the observation of a realized bet. Quite naturally, we establish below that the bets are more informative when individual bettors possess better information, in the sense of Blackwell. We find that the FLB arises for any market probability, provided that bets are sufficiently informative, and that it is more pronounced with greater informativeness.

Proposition 5 Assume $K=2$ and that the belief distribution is symmetric. (i) If individual bettors are better informed, bets are more informative. (ii) Take as given any longshot's market probability $\pi_{k}<1 / 2$ (respectively, a favorite's $\pi_{k}>1 / 2$ ). The associated posterior probability $\beta_{k}$ is decreasing (respectively, increasing) in the bets' informativeness, and is strictly smaller (respectively, greater) than $\pi_{k}$ if and only if

$$
\frac{1}{1-2 \pi_{k}} \log \left(\frac{1-\pi_{k}}{\pi_{k}}\right)<N \log \left(\frac{\eta(1 \mid 1)}{\eta(1 \mid 2)}\right)
$$

To illustrate the impact of private information on the FLB, consider a special case of the Dirichlet signal example (developed in detail in Appendix B) with $K=2$ outcomes. ${ }^{25}$ In this example, bettors have less private information when $\theta$ is higher and are completely uninformed in the limit as $\theta \rightarrow \infty$.

\footnotetext{
${ }^{25}$ In a model without private information, Brown and Lin (2003) consider Dirichlet distributed prior beliefs. Our setting with Dirichlet distributed posterior beliefs is fundamentally different. See footnote 12 .
} 


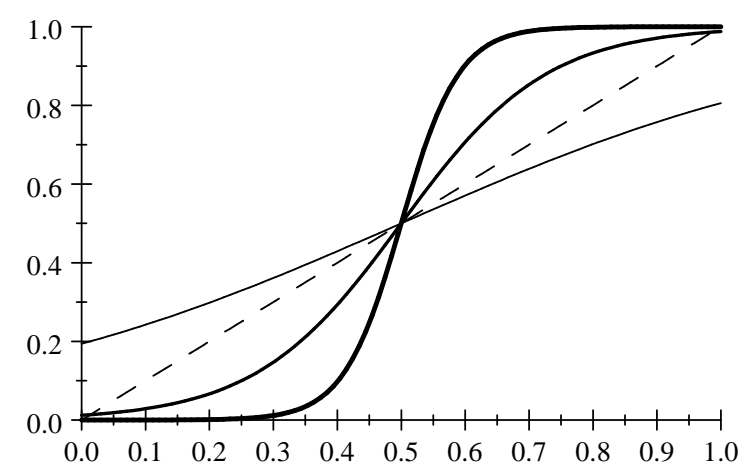

Figure 1: Posterior Probability as a Function of market probability, For $\theta=10,1,1 / 10$

Lemma 1 In the Dirichlet signal example for $K=2$ and any real $\theta>0$, the equilibrium probability that any bettor bets on the winner is

$$
\eta(k \mid k)=\frac{1}{2}+\frac{\Gamma(2 \theta+1)}{\Gamma(\theta+1) \Gamma(\theta)} \frac{4^{-\theta}}{2 \theta} .
$$

Holding fixed the number of bettors at $N=4$, Figure 1 displays the posterior probability (2) as a function of the market probability, $\pi$, for three levels of the Dirichlet parameter $\theta=10,1,1 / 10$ (corresponding to equilibrium probabilities $\eta(k \mid k)=.588, .75, .942$ ), represented in progressively darker shading. For an given level of $\theta$ and therefore $\eta(k \mid k)$, the FLB inequality (4) is harder to satisfy at more extreme market probabilities. At the easiest point, $\pi_{1}=1 / 2$, the condition is $2<N \log [\eta(1 \mid 1) / \eta(1 \mid 2)]$. Hence, there is a reverse FLB when the signal is very noisy. As the signal's informativeness rises above a critical value, the FLB occurs in an ever larger region around $\pi_{1}=1-\pi_{2}=1 / 2$. As the figure illustrates, the FLB arises for any market probability provided that the signal is sufficiently informative.

\section{$5.2 \quad$ Number of Bettors}

How does the FLB depend on the number of bettors, $N$ ? Because each bettor draws an i.i.d. signal from the same distribution, increasing the number of informed bettors increases the amount of information.

Proposition 6 Assume that the belief distribution is symmetric. Take as given any longshot's market probability $\pi_{k}<1 / 2$ (respectively, a favorite's $\pi_{k}>1 / 2$ ). The associated 


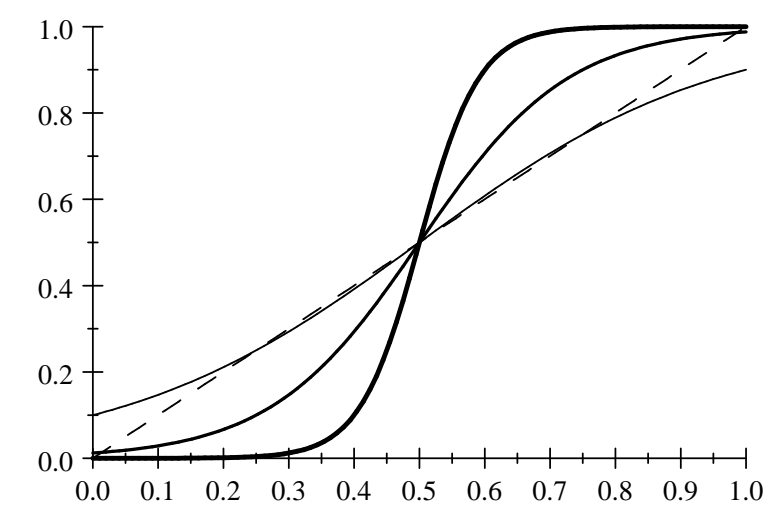

Figure 2: Posterior Probability as a Function of MARKet Probability, For $\mathrm{N}=2,4,10$

posterior probability $\beta_{k}$ is decreasing (respectively, increasing) in the number $N$ of informed bettors.

The more bettors, the greater the extent of the FLB. ${ }^{26}$ To illustrate, consider the uniform signal example developed in Appendix B, corresponding to the Dirichlet example with $\theta=1$. For the special case with $K=2$, we have $\eta(1 \mid 1)=\eta(2 \mid 2)=3 / 4$ from (2). Figure 2 shows the posterior probability $\beta_{k}$ as a function of the market probability $\pi_{k}=$ $n / N$. The curves correspond to $N=2,4$, and 10, drawn in progressively thicker shading. The FLB results if the posterior probability is below the (dashed) diagonal for $\pi<1 / 2$ and above the diagonal for $\pi>1 / 2$.

\subsection{Number of Outcomes}

To derive results about changes in the number of outcomes, we need again to impose some structure on the distribution of beliefs. We begin by considering completely uninformed bettors, then turn to the uniform signal example, and finally extend the model to allow bets on more than one outcome.

Reverse Bias in Lotto. Unless the game is corrupt, Lotto gamblers have no private information about the outcome drawn, regardless of the number of possible combinations.

\footnotetext{
${ }^{26}$ In addition, the number of bettors also affects the probability distribution over $\pi$. The law of large numbers implies that a greater number of bettors will generate a less random $\pi$. As the number of bettors approaches infinity, the realization of market probabilities conditional on outcome $k$ becomes deterministic and fully reveals the outcome.
} 
Lotto corresponds to a degenerate version of our model with completely uninformative signals. In equilibrium, each gambler picks a random combination with probability $1 / K$. When $N / K$ tends to infinity, the distribution of bets across all outcomes becomes uniform, according to the law of large numbers. However, the designers of Lotto games typically set $K$ according to the rule of thumb $K \approx N$ (see, e.g., Ian Walker and Juliet Young, 2001). Hence, there remains a fair amount of noise in the bet distribution.

As a result of this noise, some combinations receive no bets, others receive one bet, and still others receive two or more bets. The market probability of a Lotto outcome would again be the fraction of bets placed on it. We show here that outcomes with high market probability have low expected return. Note that the jackpot is shared among all those who picked the winning combination and that the posterior probability is equal to the prior probability, $1 / K$, regardless of the market probability. The expected return to a bet on an outcome with market probability $\pi$ is thus $(1-\tau) /(\pi K)-1$. Because this return is decreasing in the market probability, the reverse FLB always results. This bias is an immediate consequence of how parimutuel payoffs are determined.

Next, we show that when $K$ is large and $N$ is fixed, an increase in $K$ results in a further increase in the reverse FLB. Holding $N$ fixed, as $K$ increases (i.e., as $N / K$ tends to zero), all gamblers will bet on different outcomes with probability 1. A favorite then has market probability $1 / N$, while the posterior (as well as prior) probability of that outcome is $1 / K$. The reverse FLB, measured by the expected loss to a bet on any favorite, $1-(1-\tau) N / K$, is increasing in $K$.

Reduced Bias in Exotic Bets. We now turn to consider how the FLB depends on the number of outcomes, $K$, holding the number of bettors fixed. We restrict attention to the uniform signal example, for which we can obtain an explicit characterization of the equilibrium conditional betting probabilities:

Lemma 2 In the uniform signal example, the equilibrium probability that any bettor bets on the winner is

$$
\eta(k \mid k)=\sum_{j=0}^{K-1}\left(\begin{array}{c}
K-1 \\
j
\end{array}\right) \frac{(-1)^{j}}{(j+1)^{2}} .
$$

Within the uniform signal example, we can use (6) to compute the main statistics of the equilibrium for different values of $K$, as reported in Figure 3. Note that the likelihood ratio 


\begin{tabular}{|l||ccccc|}
\hline & $K=2$ & $K=5$ & $K=10$ & $K=100$ & $K=10,000$ \\
\hline \hline$\eta(k \mid k)$ & $3 / 4$ & 0.457 & 0.293 & $5.19 \times 10^{-2}$ & $9.79 \times 10^{-4}$ \\
$\eta(k \mid l)$ & $1 / 4$ & 0.136 & 0.0786 & $9.48 \times 10^{-3}$ & $9.99 \times 10^{-5}$ \\
$\frac{\eta(k \mid k)}{\eta(k \mid l)}$ & 3 & 3.36 & 3.73 & 5.47 & 9.80 \\
$\pi^{*}$ & $1 / 2$ & 0.277 & 0.161 & $2.51 \times 10^{-2}$ & $3.86 \times 10^{-4}$ \\
\hline
\end{tabular}

Figure 3: DePendence of EQUilibrium structure on number of outcomes $K$

for updating has the following properties. When the number of outcomes rises, observation of a bet on outcome $k$ contains more favorable information for outcome $k$. In Figure $3, \pi^{*}$ from (3) is the market belief that is neutral when $N \rightarrow \infty$. When $K=2$, an outcome earns a positive expected monetary return as soon as the market probability is higher than the prior, $1 / 2$. However, for greater $K$, we note that the neutral market probability satisfies $\pi^{*}>1 / K$. When there are many possible winning outcomes, any particular outcome must attract more than the average fraction $1 / K$ of total bets for the posterior probability to be above the market probability. Intuitively, the noise present in the signals used by bettors induces this wedge between the prior probability and the market probability above which a horse becomes a good bet.

When the number of outcomes is large relative to the number of bettors, the noise effect dominates in equation (2), resulting in a reverse FLB: the posterior probability is higher than the market probability for longshots, but is lower for favorites. This finding is consistent with Peter Asch and Quandt's (1987 and 1988) observation of a reduced (or reverse) FLB for exotic bets, in which the ratio of outcomes to bettors is relatively higher than for the win pool.

Divisibility of Bets. We illustrate the role of our indivisibility assumption through two simple examples. Consider first the case without private information, with $K$ equally likely outcomes. If bets are perfectly divisible, then in the symmetric equilibrium each bettor places $1 / K$ on each outcome. As a result, the market probability is always equal to the empirical probability. The reverse FLB then disappears completely! Intuitively, our previous explanation for the reverse FLB relied on the realized bets going beyond the implied probability, but bettors can reduce this problem when bets are divisible. In reality, however, the divisibility constraint binds because the minimum bet is bounded by the price of an individual Lotto ticket. Because it is impossible to bet infinitesimal amounts and 
$N / K$ is small, the substantial amount of noise that is present in Lotto drives the reverse FLB.

Second, the incentive to bet on more than one outcome exists also when bettors are privately informed. However, this incentive is reduced because it entails acting against one's private information. To illustrate this point, we turn to an example with binary signals. Assume that there are $K=2$ equally likely outcomes, $N=2$ bettors, and no abstention. Suppose that each bettor observes a binary symmetric signal with precision $\pi=\operatorname{Pr}(s=k)>1 / 2$ and is allowed to split the bet between the two outcomes. In the symmetric equilibrium, each bettor places the fraction $y^{*} \in(0,1)$ on the outcome their signal indicates. The expected payoff of a bettor placing $y$ on the outcome believed to be most likely is

$$
\pi\left[\pi \frac{2 y}{y^{*}+y}+(1-\pi) \frac{2 y}{1-y^{*}+y}\right]+(1-\pi)\left[\pi \frac{2(1-y)}{y^{*}+1-y}+(1-\pi) \frac{2(1-y)}{1-y^{*}+1-y}\right] .
$$

In symmetric equilibrium, $y^{*}$ maximizes this expression. It is straightforward to verify that the solution is $y^{*} \in\left(\pi, \pi^{2} /\left[\pi^{2}+(1-\pi)^{2}\right]\right)$. The FLB follows. When the bettors obtain opposite signals, market and posterior probabilities are both equal to $1 / 2$. When the bettors obtain the same signal, the market probability for the favorite is $y^{*}$, while the posterior probability is $\pi^{2} /\left[\pi^{2}+(1-\pi)^{2}\right]>y^{*}$.

Intuitively, if players can bet on more than one outcome, then the amount of noise relative to information present in equilibrium decreases and the FLB increases. More generally, we expect that the incentive to bet on multiple outcomes is particularly important in exotic bets, where the ratio of outcomes to bettors is high. For example, someone who received a tip on the outcome of two races fears the noise on the outcomes of the other four races, making a "pick six" gamble (see footnote 14) akin to a lottery. Consistent with popular advice, the best strategy is to bet on many combinations involving different outcomes in those other races.

\subsection{Common Error}

Thus far we have maintained the hypothesis that the private signals are independent, conditional on the true outcome, $k$. However, there is often an unpredictable component in the outcome of the race - all the more so when weather and terrain conditions at the racetrack are uncertain. Equivalently, there is residual uncertainty about the outcome that 
could not be resolved even if infinitely many private signals were observed. To analyze the effect of common error, we define a new random variable $x \in\{1, \ldots, K\}$ as the information state and set at $\operatorname{Pr}(x=k \mid k)=\sigma>1 / K$ the chance that the information state is identical to the true outcome. Knowing information state $x$, the posterior probability of the outcome $k=x$ is then $\sigma$. Individual signals are informative about $x$, but carry no further information about the outcome.

By the symmetry of this setup, it continues to be a symmetric equilibrium for each individual to bet on the most likely outcome according to their private belief. Denote by $\tilde{\eta}(l \mid x)$ the chance of an individual betting on $l$ in information state $x$. Generalizing (2), an outcome $k$ with market probability $\pi$ now has posterior probability

$$
\tilde{\beta}=\frac{\sigma \tilde{\eta}(k \mid k)^{N \pi}[1-\tilde{\eta}(k \mid k)]^{N(1-\pi)}+(1-\sigma) \tilde{\eta}(k \mid x)^{N \pi}[1-\tilde{\eta}(k \mid x)]^{N(1-\pi)}}{\tilde{\eta}(k \mid k)^{N \pi}[1-\tilde{\eta}(k \mid k)]^{N(1-\pi)}+(K+1-2 \sigma) \tilde{\eta}(k \mid x)^{N \pi}[1-\tilde{\eta}(k \mid x)]^{N(1-\pi)}},
$$

where $x \neq k$. In this symmetric setting, the expected return associated with market

probability $\pi$ is $\tilde{\beta}(1-\tau) / \pi-1$. By simple algebra, it can be verified that this expected return is an increasing function of $\sigma$ when $\pi>\pi^{*}$. Hence, an increase in ex post noise (i.e., a reduction of $\sigma$ ) flattens the expected return curve for favorites. As Snowberg and Wolfers (2005) document, expected returns in horse races are an increasing function of the market probability, but present a flat segment for intermediate market probabilities. We conclude that the realistic addition of ex post noise brings our model closer to explaining this occurrence of a flat segment.

\subsection{Participation}

Turn to the second case of Proposition 2, in which some, but not all bettors choose to abstain. This extension allows us to show that the FLB unambiguously results as the notrade outcome is approached. To simplify the exposition, focus on a setting with $K=2$ outcomes and suppose that the symmetric belief distribution has full support on $[0,1]$. In equilibrium, bettors with strong beliefs $p>\hat{p}_{1}$ in favor of outcome 1 bet on outcome 1 , while those with strong beliefs $p<\hat{p}_{2}$ (with $\hat{p}_{2}<\hat{p}_{1}$ ) bet on 2 .

Participation decreases when the recreational value of betting, $u$, is reduced (or, equivalently, the takeout rate, $\tau$, is increased), because the expected loss from betting is not compensated by the recreational value for more bettors with intermediate beliefs. Hence, the overall amount of information present in the market is also reduced. However, the bet- 
tors with intermediate private beliefs are the first to drop out. Thus, the realized bets will contain relatively more information and less noise. According to the logic of Proposition 5, more informative bets contribute to the FLB.

Proposition 7 Assume that $K=2$, that the belief distribution is symmetric and unbounded, and that $u \in\left(\tau, u^{*(N)}\right)$. (i) There exists a symmetric equilibrium with $\hat{p}_{2}=$ $1-\hat{p}_{1} \in(0,1 / 2)$. (ii) Take as given any bet realization with total amounts $b_{1}, b_{2}>0$ placed on the two outcomes. If $u$ is sufficiently close to $\tau$, a longshot's market probability $\pi_{1}=b_{1} /\left(b_{1}+b_{2}\right)<1 / 2$ (respectively, a favorite's $\pi_{1}>1 / 2$ ) is strictly greater (respectively, smaller) than the associated posterior probability $\beta_{1}$.

Note that if there is a reverse FLB at $u^{*}$, it will persist as $u$ falls slightly below $u^{*}$ because the equilibrium changes continuously. However, the reverse bias is overturned as $u$ falls further towards $\tau$, according to Proposition 7. In conclusion, information swamps noise as trade vanishes (and the outcome of the no-trade theorem is approached) with the reduction in recreational value, resulting unambiguously in the FLB.

\subsection{Ex Ante Asymmetries}

We turn to the effect of ex ante asymmetries in the prior probabilities of different outcomes. Even though races are designed to be balanced, typically the public has access to a fair amount of prior information, in the form of past performance, morning line odds, and handicappers' picks. When computing expected returns associated to market probabilities, empiricists typically do not have access to the prior probabilities of different outcomes. This lack of ex ante information about an outcome's prior probability results in a mixing effect: an ex post favorite may have already been an ex ante favorite (having received disappointingly few bets) or an ex ante longshot (with surprisingly many informed bets). As a result, the empirical methodology used to compute expected returns induces the expected returns for intermediate market probabilities to be relatively flat with respect to changes in market probabilities.

Using the prior belief distribution and the equilibrium conditional probabilities $\eta(m \mid l)$, the expected return associated with any given realized market probability can be computed as follows: 


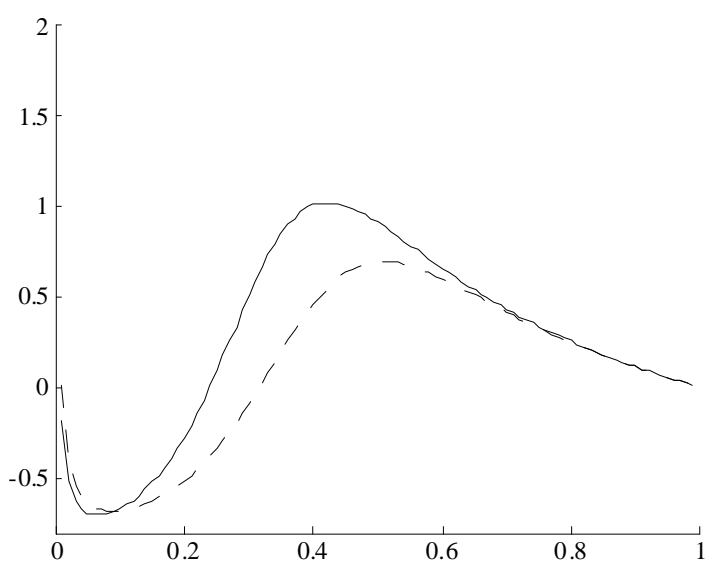

Figure 4: EXPECTED RETURN AS A FUNCTION OF MARKET PROBABILITY, DEPENDING ON SYMMETRY IN PRIOR BELIEFS

Proposition 8 With $N$ active bettors, the expected return corresponding to market probability $\pi$ is

$$
\bar{R}_{\pi}=\frac{1-\tau}{\pi} \frac{\sum_{k=1}^{K} q_{k} \eta(k \mid k)^{N \pi}(1-\eta(k \mid k))^{N(1-\pi)}}{\sum_{m=1}^{K} \sum_{l=1}^{K} q_{l} \eta(m \mid l)^{N \pi}(1-\eta(m \mid l))^{N(1-\pi)}}-1
$$

We illustrate the effect of ex ante asymmetries on expected returns in the context of the uniform signal example introduced in Appendix B. For the numerical illustration, let $\tau=0$ and set $u$ sufficiently large to ensure full participation.

Lemma 3 In the uniform signal example, given $W_{1}, \ldots, W_{K}$, the best response of each bettor implies for every $l \neq k$,

$$
\begin{aligned}
\eta(k \mid k)= & \sum_{j=0}^{K-1} \sum_{A \subseteq\{1, \ldots, K\} \backslash k,|A|=j} \frac{(-1)^{j}}{\left(1+\sum_{m \in A} \frac{q_{k} W_{k}}{q_{m} W_{m}}\right)^{2}}, \\
\eta(l \mid k)= & \sum_{j=0}^{K-1} \sum_{A \subseteq\{1, \ldots, K\} \backslash k,|A|=j} \frac{(-1)^{j}}{1+\sum_{m \in A} \frac{q_{k} W_{k}}{q_{m} W_{m}}} \\
& +\sum_{j=0}^{K-2} \sum_{A \subseteq\{1, \ldots, K\} \backslash\{k, l\},|A|=j} \frac{(-1)^{j} q_{k} W_{k}}{q_{l} W_{l}\left(1+\frac{q_{k} W_{k}}{q_{l} W_{l}}+\sum_{m \in A} \frac{q_{k} W_{k}}{q_{m} W_{m}}\right)^{2}} .
\end{aligned}
$$

From Proposition 1, with full participation we have $W_{k}=\left[1-(1-\eta(k \mid k))^{N}\right] / \eta(k \mid k)$. This equation and (8) reduces finding the equilibrium to a matter of solving equations. 
Once the equilibrium values of $W_{1}, \ldots, W_{K}$ have been determined as a solution to these equations, (8) and (9) allow us to calculate the equilibrium betting probabilities, which are then used to compute the expected return (7).

To visualize the effect of race asymmetry, Figure 4 displays how the expected return (7) for different market probabilities varies with the prior probability distribution for an example with $N=10$ bettors and $K=9$ outcomes. The solid line refers to the symmetric case with $q_{k}=1 / 9$ for every outcome $k$, while the dashed line refers to the asymmetric prior $q=(0.29,0.21,0.17,0.13,0.09,0.05,0.03,0.02,0.01)$, a specification proposed by Chadha and Quandt (1996). ${ }^{27}$ For the asymmetric race, the return tends to be closer to zero for most odds, as empirically observed. Intuitively, intermediate market probabilities can arise in two ways in an asymmetric race. Either the ex ante favorite has received relatively few bets, and therefore yields a negative return by the informational FLB, or the ex ante longshot has received relatively many bets, and thus yields a positive return. These two effects counteract each other and tend to flatten the expected return curve in the intermediate range. This observation improves our model's fit to the main qualitative features of expected returns observed in horse races, as documented by Erik Snowberg and Wolfers (2005).

\section{Comparison with Other Explanations}

In this section, we compare the performance of our theory with the main alternative explanations for the FLB in parimutuel betting markets. ${ }^{28}$ The most notable alternative theories that have been proposed in the literature are the following: (1) Griffith (1949) suggested that the FLB is due to a tendency of individual bettors to overestimate low probability events; (2) Isaacs (1953) noted that an informed monopolist bettor who can place multiple bets does not set the expected return on his marginal bet at zero, because this destroys the return on inframarginal bets; (3) Weitzman (1965) hypothesized that individual bettors are risk loving, and thus willing to accept a lower expected payoff when they bet on riskier longshots; ${ }^{29}$ (4) Ali (1977) showed that if bettors have heterogeneous

\footnotetext{
${ }^{27}$ We have verified in simulations that the properties displayed in this picture hold more generally.

${ }^{28}$ We refer to Ottaviani and Sørensen (2008) for a more detailed presentation of the theoretical explanations for the FLB that have been proposed in the literature.

${ }^{29}$ See also Richard N. Rosett (1965), Quandt (1976), and Ali (1977) on the risk-loving explanation. According to Joseph Golec and Maurry Tamarkin (1998), the bias is compatible with preferences for
} 
(prior) beliefs, then the market probability of the favorite is lower than the median bettor's belief. The FLB then results if the belief of the median bettor is correct; (5) William Hurley and Lawrence McDonough (1995) and Dek Terrell and Amy Farmer (1996) showed that the FLB can result because the amount of arbitrage is limited by the track take.

While we believe that all of these theories can contribute to explain the evidence, the information-based theory developed in this paper has a number of merits:

- Our theory builds on the realistic assumption that the differences in beliefs among bettors are generated by private information (see e.g. Nicholas F.R. Crafts 1985). Only by modeling the informational determinants of beliefs explicitly, can we address the natural question of information aggregation. ${ }^{30}$

- Our theory offers a parsimonious explanation for the FLB and its reverse. Our theory also predicts that the FLB is lower, or reversed, when the number of bettors is low (relative to the number of outcomes), as Russell S. Sobel and S. Travis Raines (2003) and Marshall Gramm and Douglas H. Owens (2005) verify empirically. ${ }^{31}$

- Our theory is compatible with the reduced level of FLB that Asch and Quandt (1987 and 1988) document in exotic bets, such as exactas and trifectas (see also footnote 14). ${ }^{32}$ Asch and Quandt (1988) conclude in favor of private information because the payoffs on winners tend to be more depressed in the exacta than in the win pool. ${ }^{33}$

- Our explanation can account for the pattern of expected returns that Snowberg and Wolfers (2005) document. Asymmetries in the prior probabilities of the different outcomes and common errors in the bettors' beliefs tend to flatten the expected

skewness rather than risk. Jullien and Salanié (2000) use data from fixed-odds markets to argue in favor of non-expected utility models.

${ }^{30}$ See Ottaviani and Sørensen (2005a) on the interplay of heterogeneous priors and private information in prediction markets.

${ }^{31}$ The preponderance of noise might also account for some of Andrew Metrick's (1996) findings in basketball betting.

${ }^{32}$ Risk loving does not seem compatible with arbitrage across exacta and win pools. As Snowberg and Wolfers (2005) stress, probability weighting would need to be combined with additional misperceptions to be compatible with arbitrage across betting pools.

${ }^{33}$ Asch and Quandt (1988) observe that the market probabilities recovered from the win pool overestimate the market probabilities on the exacta pool by a much larger margin for winning than for losing horses. 
returns for intermediate market probabilities. ${ }^{34}$

- Our theory is compatible with the fact that late bets tend to contain more information about the horses' finishing order than earlier bets, as Asch, Burton G. Malkiel, and Quandt (1982) observe. Ottaviani and Sørensen (2006) demonstrate that late informed betting will result in equilibrium when (many small) bettors are allowed to optimally time their bets.

- Theories based on private information can explain the occurrence of the FLB in both fixed-odds and parimutuel markets, as well as the lower level of bias observed in parimutuel markets, as Alistair C. Bruce and Johnnie E.V. Johnson (2001) among others document (see footnote 13 above).

\section{Conclusion}

This paper investigates the information aggregation properties of parimutuel markets. The sign and the extent of the FLB depend on the amount of information relative to noise that is present in the market. When there is little private information, posterior odds are close to prior odds, even when the market odds are extreme. In this case, deviations of market odds from prior odds are mostly due to the noise contained in the signal. Systematically, market odds are more extreme than posterior odds, and the reverse FLB results.

As the number of bettors increases, the realized market odds contain more information and less noise. Holding fixed the level of market odds, the corresponding posterior odds are then more extreme, increasing the extent of the FLB. Note that the FLB always arises with a large number of bettors, provided that they have some private information. This is confirmed by Ottaviani and Sørensen (2006) in a model with a continuum of privately informed bettors. In that setting there is no noise, so that the FLB always results.

Our theory delivers a number of comparative statics predictions. The FLB is more pronounced when the number of (informed) bettors increases, bettors have more private information, the number of outcomes decreases, the recreational value of the event de-

\footnotetext{
${ }^{34}$ The observed flat segment is theoretically compatible with risk loving or probability weighting, but would imply a very specific preference pattern. For example, if bettors had mean-variance preferences, as posited by Quandt (1986), then the expected return would be strictly increasing in the market probability. Limited arbitrage à la Hurley and McDonough (1995) can explain the flat segment for horses with probability above a certain threshold, but not the decreasing segment for strong favorites.
} 
creases, or the takeout rate increases. In addition, the flat segment in expected returns for intermediate odds can be explained by introducing a realistic level of asymmetry in the prior belief distribution or a common error component in the information of bettors.

These predictions shed light on the available evidence and could be tested by exploiting the variation across betting environments. The amount of private information tends to vary consistently depending on the prominence of the underlying event. Similarly, the amount of noise present depends on the number of outcomes, as well as on the observability of past bets. For example, there is a sizeable amount of noise in lotteries and exotic bets, because the number of outcomes is high relative to the number of tickets sold and the opponents' bets are not observed. 


\section{References}

Ali, Mukhtar M. 1977. "Probability and Utility Estimates for Racetrack Bettors." Journal of Political Economy, 85(4): 803-815.

Asch, Peter, Burton G. Malkiel, and Richard E. Quandt. 1982. "Racetrack Betting and Informed Behavior." Journal of Financial Economics, 10(2): 187-194.

Asch, Peter, and Richard E. Quandt. 1987. "Efficiency and Profitability in Exotic Bets." Economica, 54(215): 289-298.

Asch, Peter, and Richard E. Quandt. 1988. "Betting Bias in Exotic Bets." Economics Letters, 28(3): 215-219.

Borel, Émile. 1938. "Sur le Pari Mutuel." Comptes Rendus Hebdomadaires des Séances de l'Académie des Sciences, 207(3): 197-200.

Brown, Lawrence D., and Yi Lin. 2003. "Racetrack Betting and Consensus of Subjective Probabilities." Statistics and Probability Letters, 62(2): 175-187.

Bruce, Alistair C., and Johnnie E.V. Johnson. 2001. "Efficiency Characteristics of a Market for State Contingent Claims." Applied Economics, 33(13): 1751-1754.

Busche, Kelly, and Christopher D. Hall. 1988. "An Exception to the Risk Preference Anomaly." Journal of Business, 61(3): 337-346.

Chadha, Sumir, and Richard E. Quandt. 1996. "Betting Bias and Market Equilibrium in Racetrack Betting." Applied Financial Economics, 6(3): 287-292.

Conlisk, John. 1993. "The Utility of Gambling." Journal of Risk and Uncertainty, 6(3): 255-275.

Crafts, Nicholas F.R. 1985. "Some Evidence of Insider Knowledge in Horse Race Betting in Britain." Economica, 52(27): 295-304.

Economides, Nicholas, and Jeffrey Lange. 2005. "A Parimutuel Market Microstructure for Contingent Claims Trading." European Financial Management, 11(1): 25-49.

Eisenberg, Edmund, and David Gale. 1959. "Consensus of Subjective Probabilities: The Pari-Mutuel Method." Annals of Mathematical Statistics, 30(1): 165-168.

Forrest, David, O. David Gulley, and Robert Simmons. 2000. "Testing for Rational Expectations in the UK National Lottery." Applied Economics, 32(3): 315326.

Golec, Joseph, and Maurry Tamarkin. 1998. "Bettors Love Skewness, Not Risk, at the Horse Track." Journal of Political Economy, 106(1): 205-225.

Gramm, Marshall, and Douglas H. Owens. 2005. "Determinants of Betting Market Efficiency." Applied Economics Letters, 12(3): 181-185. 
Griffith, Richard M. 1949. "Odds Adjustment by American Horse-Race Bettors." American Journal of Psychology, 62(2): 290-294.

Hausch, Donald B., and William T. Ziemba. 1995. "Efficiency of Sports and Lottery Betting Markets." In Handbook of Operations Research and Management Science, Volume 9, ed. Robert A. Jarrow, Vojislav Maksimovic, and William T. Ziemba, 545-580. Amsterdam: Elsevier North Holland.

Hurley, William, and Lawrence McDonough. 1995. "Note on the Hayek Hypothesis and the Favorite-Longshot Bias in Parimutuel Betting." American Economic Review, 85(4): 949-955.

Isaacs, Rufus. 1953. "Optimal Horse Race Bets." American Mathematical Monthly, 60(5): 310-315.

Johnson, Norman L., and Samuel Kotz. 1972. Distributions in Statistics: Continuous Multivariate Distributions. New York: John Wiley \& Sons.

Jullien, Bruno, and Bernard Salanié. 2000. "Estimating Preferences under Risk: The Case of Racetrack Bettors." Journal of Political Economy, 108(3): 503-530.

Jullien, Bruno, and Bernard Salanié. 2008. "Empirical Evidence on the Preferences of Racetrack Bettors." In Handbook of Sports and Lottery Markets, ed. William T. Ziemba and Donald B Hausch, 27-49. Amsterdam: North Holland.

Koessler, Frédéric, Charles Noussair, and Anthony Ziegelmeyer. 2008. "Parimutuel Betting under Asymmetric Information." Journal of Mathematical Economics, 44(7): 733-744.

Levitt, Steven D. 2004. "Why Are Gambling Markets Organised So Differently from Financial Markets?" Economic Journal, 114(495): 223-246.

Metrick, Andrew. 1996. "March Madness? Strategic Behavior in NCAA Basketball Tournament Betting Pools." Journal of Economic Behavior and Organization, 30(2): $159-172$.

Milgrom, Paul, and Nancy Stokey. 1982. "Information, Trade and Common Knowledge." Journal of Economic Theory, 26(1): 17-27.

National Thoroughbred Racing Association: Wagering Systems Task Force. 2004. Declining Purses and Track Commissions in Thoroughbred Racing: Causes and Solutions. http://www.hbpa.org/resources/Ntrataskreportsep04.pdf.

Ottaviani, Marco, and Peter N. Sørensen. 2005a. "Aggregation of Information and Beliefs in Prediction Markets." Unpublished.

Ottaviani, Marco, and Peter N. Sørensen. 2005b. "Parimutuel versus Fixed-Odds Markets." Unpublished. 
Ottaviani, Marco, and Peter N. Sørensen. 2006. "The Timing of Parimutuel Bets." Unpublished.

Ottaviani, Marco, and Peter N. Sørensen. 2008. "The Favorite-Longshot Bias: An Overview of the Main Explanations." In Handbook of Sports and Lottery Markets, ed. William T. Ziemba and Donald B. Hausch, 83-101. Amsterdam: North Holland.

Ottaviani, Marco, and Peter N. Sørensen. Forthcoming. "Surprised by the Parimutuel Odds?" American Economic Review.

Quandt, Richard E. 1986. "Betting and Equilibrium." Quarterly Journal of Economics, 101(1): 201-208.

Rosett, Richard N. 1965. "Gambling and Rationality." Journal of Political Economy, 73(6): 595-607.

Sauer, Raymond D. 1998. "The Economics of Wagering Markets." Journal of Economic Literature, 36(4): 2021-2064.

Shin, Hyun Song. 1991. "Optimal Betting Odds Against Insider Traders." Economic Journal, 101(408): 1179-1185.

Shin, Hyun Song. 1992. "Prices of State Contingent Claims with Insider Traders, and the Favourite-Longshot Bias." Economic Journal, 102(411): 426-435.

Snowberg, Erik, and Justin Wolfers. 2005. "Explaining the Favorite-Longshot Bias: Is It Risk-Love or Misperceptions?" Unpublished.

Sobel, Russell S., and S. Travis Raines. 2003. "An Examination of the Empirical Derivatives of the Favourite-Longshot Bias in Racetrack Betting." Applied Economics, 35(4): 371-385.

Terrell, Dek, and Amy Farmer. 1996. "Optimal Betting and Efficiency in Parimutuel Betting Markets with Information Costs." Economic Journal, 106(437): 846-868.

Thaler, Richard H., and William T. Ziemba. 1988. "Parimutuel Betting Markets: Racetracks and Lotteries." Journal of Economic Perspectives, 2(2): 161-174.

Vaughan Williams, Leighton, and David Paton. 1998. "Why Are Some FavouriteLongshot Biases Positive and Others Negative?" Applied Economics, 30(11): 15051510 .

Walker, Ian, and Juliet Young. 2001. "An Economist's Guide to Lottery Design." Economic Journal, 111(475): F700-722.

Weitzman, Martin. 1965. "Utility Analysis and Group Behavior: An Empirical Analysis." Journal of Political Economy, 73(1): 18-26.

Wolfers, Justin, and Eric Zitzewitz. 2004. "Interpreting Prediction Market Prices as Probabilities." Unpublished. 


\section{Appendix A: Proofs of Propositions}

Proof of Proposition 1. Suppose first that $\eta(k \mid k)=0$ so no opponent bets on $k$. A bet on $k$ then returns the expected pool net of the takeout, $(1-\tau)\left[1+(N-1) \sum_{l \neq k} \eta(l \mid k)\right]$. In the following, assume $\eta(k \mid k)>0$. If the realized number of opponent bets is $n$ and outcome $k$ is realized, the conditional chance for an active opponent to bet on outcome $k$ is $\eta(k \mid k) / \sum_{l} \eta(l \mid k)$. The conditional expected payment is then

$$
\begin{aligned}
& (1-\tau) \sum_{\hat{b}_{k}=0}^{n} \frac{n+1}{\hat{b}_{k}+1}\left(\begin{array}{c}
n \\
\hat{b}_{k}
\end{array}\right)\left(\frac{\eta(k \mid k)}{\sum_{l} \eta(l \mid k)}\right)^{\hat{b}_{k}}\left(1-\frac{\eta(k \mid k)}{\sum_{l} \eta(l \mid k)}\right)^{n-\hat{b}_{k}} \\
= & \frac{(1-\tau)}{\eta(k \mid k) / \sum_{l} \eta(l \mid k)} \sum_{b_{k}=1}^{n+1}\left(\begin{array}{c}
n+1 \\
b_{k}
\end{array}\right)\left(\frac{\eta(k \mid k)}{\sum_{l} \eta(l \mid k)}\right)^{b_{k}}\left(1-\frac{\eta(k \mid k)}{\sum_{l} \eta(l \mid k)}\right)^{n+1-b_{k}} \\
= & (1-\tau) \frac{1-\left[1-\eta(k \mid k) / \sum_{l} \eta(l \mid k)\right]^{n+1}}{\eta(k \mid k) / \sum_{l} \eta(l \mid k)} .
\end{aligned}
$$

Because $n$ is binomially distributed with parameter $\sum_{l} \eta(l \mid k)$, the desired expression for $W_{k} /(1-\tau)$ is

$$
\begin{aligned}
& \sum_{n=0}^{N-1} \frac{1-\left[1-\eta(k \mid k) / \sum_{l} \eta(l \mid k)\right]^{n+1}}{\eta(k \mid k) / \sum_{l} \eta(l \mid k)}\left(\begin{array}{c}
N-1 \\
n
\end{array}\right)\left(\sum_{l} \eta(l \mid k)\right)^{n}\left(1-\sum_{l} \eta(l \mid k)\right)^{N-1-n} \\
= & \frac{\sum_{l} \eta(l \mid k)}{\eta(k \mid k)}-\frac{\sum_{l \neq k} \eta(l \mid k)}{\eta(k \mid k)} \sum_{n=0}^{N-1}\left(\begin{array}{c}
N-1 \\
n
\end{array}\right)\left(\sum_{l \neq k} \eta(l \mid k)\right)^{n}\left(1-\sum_{l} \eta(l \mid k)\right)^{N-1-n} \\
= & \frac{\sum_{l} \eta(l \mid k)-\left[\sum_{l \neq k} \eta(l \mid k)\right][1-\eta(k \mid k)]^{N-1}}{\eta(k \mid k)} .
\end{aligned}
$$

Proof of Proposition 2. First, suppose that $u>\tau$, but no one is betting. Individuals with private beliefs $p_{k}$ sufficiently close to 1 gain from deviating to a bet on outcome $k$, because the expected utility from doing so is arbitrarily close to $(1-\tau)-1+u>0$.

Existence of a symmetric equilibrium follows from a standard fixed point argument. Note that the winner's payoffs $\left(W_{1}, \ldots, W_{K}\right)$ lie in the compact, convex box $[1, N]^{K}$. Given any vector $W$ from this box, the best response of a bettor consists (up to a set of probability zero) in betting on the outcome $k$ with the greatest corresponding $p_{k} W_{k}$, or abstaining if this greatest value is negative. Given the strategy of the bettor, we can deduce the positive probability with which bets on each outcome are placed, and then calculate the expected payoffs. This uniquely defined map from $[1, N]^{K}$ into itself is continuous, and hence has a 
fixed point by Brouwer's theorem. The strategy associated with the fixed point constitutes a symmetric equilibrium strategy.

In any symmetric equilibrium where everyone is betting, we can find a belief $\hat{p}$ at which the interim expected utility is at the lowest level. By upper hemi-continuity of the equilibrium correspondence and compactness of the strategy space, there exists an equilibrium where this lowest interim utility $U$ is, in turn, minimal. Holding fixed these equilibrium strategies, the critical value $u^{*(N)}$ is defined as the solution to $U=0$.

Finally, suppose that $u \leq \tau$. Any bet is reduced to $1-\tau$ before being placed in the pool. By the logic of the no-trade theorem, it is impossible that all active bettors expect a return in excess of $1-\tau$. If there is a positive chance of betting, some active bettors expect a return less than $1-\tau \leq 1-u$ and are better off abstaining. This contradiction implies that there can be no active bettors at all.

Proof of Proposition 3. By symmetry of the betting strategy and the belief distribution, we have $\eta(1 \mid 1)=\cdots=\eta(K \mid K)$ and hence $W_{1}=\cdots=W_{K}>0$. The expected utility from a bet on $k$ is $p_{k} W_{k}+u-1$, thus the best response is to bet on the outcome with the greatest $p_{k}$, as claimed. Again, by symmetry, $\eta(k \mid l)$ assumes the same value for every pair $k \neq l$. Given that the probabilities sum to one, the proof is complete once we show that $\eta(1 \mid 1)>1 / K$. The prior chance of outcome 1 is $1 / K$, so Bayes' rule implies that the density of beliefs satisfies $g(p \mid 1)=K p_{1} g(p)$. When $p_{1}$ is greatest, almost surely $p_{1}>1 / K$. Finally, symmetry implies that with ex ante probability $1 / K, p_{1}$ is greatest. Hence,

$$
\eta(1 \mid 1)=\int_{p \mid p_{1} \text { greatest }} g(p \mid 1) d p=\int_{p \mid p_{1} \text { greatest }} K p_{1} g(p) d p>\int_{p \mid p_{1} \text { greatest }} g(p) d p=\frac{1}{K} .
$$

Proof of Proposition 4. Let $\pi_{k}<\pi^{*}$ be given. The desired inequality is

$$
\frac{1-\pi_{k}}{\pi_{k}}<(K-1)\left(\frac{\eta(k \mid l)}{\eta(k \mid k)}\right)^{n}\left(\frac{1-\eta(k \mid l)}{1-\eta(k \mid k)}\right)^{N-n}
$$

where $l \neq k$. Take the natural logarithm, use $n / N=\pi_{k}$, and re-arrange (10) to arrive at

$$
\frac{1}{N} \log \left(\frac{1-\pi_{k}}{\pi_{k}(K-1)}\right)<\pi_{k} \log \left(\frac{\eta(k \mid l)}{\eta(k \mid k)}\right)+\left(1-\pi_{k}\right) \log \left(\frac{1-\eta(k \mid l)}{1-\eta(k \mid k)}\right) .
$$

The left-hand side tends to 0 as $N \rightarrow \infty$. The right-hand side is positive, precisely because $\pi_{k}<\pi^{*}$. 
Proof of Proposition 5. (i) Note that

$$
\eta(1 \mid 1)=\int_{1 / 2}^{1} g\left(p_{1} \mid 1\right) d p_{1}=\int_{1 / 2}^{1} 2 p_{1} g\left(p_{1}\right) d p_{1}=\int_{0}^{1 / 2}\left(1-p_{1}\right) g\left(p_{1}\right) d p_{1}+\int_{1 / 2}^{1} p_{1} g\left(p_{1}\right) d p_{1}
$$

where the second equality follows from Bayes' rule, and the third equality from the symmetry assumption. Given that the right-hand side is the expectation of a convex function of $p_{1}$, we conclude that $\eta(1 \mid 1)$ increases with a mean-preserving spread of the belief distribution. As is well known, Blackwell-better information is equivalent to such a meanpreserving spread, because in Bayesian decision problems the value function is convex in the posterior belief. Finally, $\eta(1 \mid 2)=\eta(2 \mid 1)=1-\eta(1 \mid 1)$ falls, so that the likelihood ratio $\eta(1 \mid 1) / \eta(1 \mid 2)$ increases, as desired.

(ii) With two outcomes, $\eta(2 \mid 1)=1-\eta(1 \mid 1)$. Let $\pi_{k}$ be given, and note that $\beta_{k}$ is a strictly decreasing transformation of the odds ratio

$$
\frac{1-\beta_{k}}{\beta_{k}}=\left(\frac{\eta(1 \mid 1)}{\eta(1 \mid 2)}\right)^{N\left(1-2 \pi_{k}\right)}
$$

If $\pi_{k}<1 / 2$, the odds ratio is a strictly increasing function of $\eta(1 \mid 1) / \eta(1 \mid 2)$. Taking the natural logarithm, inequality (10) reduces to (4). Because $\pi_{k}<1 / 2$ and $\eta(1 \mid 1)>\eta(1 \mid 2)$, all terms in (4) are positive. The right-hand side of (4) increases without bound when $\eta(1 \mid 1) / \eta(1 \mid 2)$ rises. The inequality is reversed if $\pi_{k}>1 / 2$.

Proof of Proposition 6. Proceeding as in (11), the natural logarithm of the odds ratio is

$$
\log \left(\frac{1-\beta_{k}}{\beta_{k}}\right)=\log (K-1)+N\left[\pi_{k} \log \left(\frac{\eta(k \mid l)}{\eta(k \mid k)}\right)+\left(1-\pi_{k}\right) \log \left(\frac{1-\eta(k \mid l)}{1-\eta(k \mid k)}\right)\right],
$$

which is monotone in $N$. It rises (respectively, falls) in $N$ when $\pi_{k}<\pi^{*}$ (respectively, $\left.\pi_{k}>\pi^{*}\right)$.

Proof of Proposition 7. (i) Suppose that all opponents use a symmetric strategy defined by the threshold $\hat{p}_{1} \in(1 / 2,1)$, with $\hat{p}_{2}=1-\hat{p}_{1}$. By symmetry of the belief distribution and the thresholds, $\eta(1 \mid 1)=\eta(2 \mid 2)$ and $\eta(1 \mid 2)=\eta(2 \mid 1)$. Hence, $W_{1}=W_{2}$. The best response of an abstaining player is, by monotonicity of the expected payoff functions, defined by the thresholds $0<\check{p}_{2}<\check{p}_{1}<1$ satisfying the indifference conditions $\check{p}_{1} W_{1}=1-u=\left(1-\check{p}_{2}\right) W_{2}$. Because $W_{1}=W_{2}$, the best response satisfies $\check{p}_{1}=1-\check{p}_{2}$. 
Now, $\hat{p}_{1}$ defines a symmetric equilibrium if the best response satisfies $\check{p}_{1}=\hat{p}_{1}$, i.e., if $\hat{p}_{1} \in(1 / 2,1)$ solves $\hat{p}_{1} W_{1}=1-u$. We argue that this equation has a solution because $u \in\left(\tau, u^{*(N)}\right)$. At $\hat{p}_{1}=1 / 2$ there would be no abstention, but then $u<u^{*(N)}$ implies $\hat{p}_{1} W_{1}<1-u$. At $\hat{p}_{1}=1$ we would obtain $\hat{p}_{1} W_{1}=W_{1} \geq 1-\tau>1-u$ by $u>\tau$. Finally, $\hat{p}_{1} W_{1}$ is a continuous function of $\hat{p}_{1}$, so there must exist some $\hat{p}_{1} \in(1 / 2,1)$ with $\hat{p}_{1} W_{1}=1-u$.

(ii) For the limit result, we first derive a bound on $W_{1}$. From (1), $W_{1} \leq(1-\tau)[\eta(1 \mid 1)+$ $\eta(2 \mid 1)] / \eta(1 \mid 1)$. Next, we consider an implication of the assumption that the belief distribution has full support. The private belief $p=\operatorname{Pr}(k=1 \mid s)$ is distributed according to a density $g$ on $[0,1]$. Bayesian updating implies that $g(p \mid 1)=2 p g(p)$ and $g(p \mid 2)=$ $2(1-p) g(p)$. Hence, $g(p \mid 1) / g(p \mid 2)=p /(1-p)$. The informativeness of the private signals implies

$$
\frac{\eta(1 \mid 2)}{\eta(1 \mid 1)}=\frac{\int_{\hat{p}_{1}}^{1} g(p \mid 2) d p}{\int_{\hat{p}_{1}}^{1} g(p \mid 1) d p}=\frac{\int_{\hat{p}_{1}}^{1} \frac{1-p}{p} g(p \mid 2) d p}{\int_{\hat{p}_{1}}^{1} g(p \mid 2) d p}<\frac{1-\hat{p}_{1}}{\hat{p}_{1}} .
$$

Given that $\eta(2 \mid 1)=\eta(1 \mid 2)$ by symmetry, these observations imply

$$
\frac{1-u}{1-\tau}=\frac{\hat{p}_{1} W_{1}}{1-\tau} \leq \hat{p}_{1}\left(1+\frac{\eta(2 \mid 1)}{\eta(1 \mid 1)}\right)<\hat{p}_{1} \frac{1}{\hat{p}_{1}}=1 .
$$

Suppose that $u \rightarrow \tau$. It follows that the inequality in (13) must vanish, from which it also follows that the inequality in (12) vanishes. It follows from inspection of inequality (12), that with full support of $p$ over $[0,1]$, this inequality can only vanish if $\hat{p}_{1} \rightarrow 1$.

Suppose now that the realized bet amounts are $b_{2}, b_{1}>0$. The implied market probability for outcome 1 is $\pi_{1}=b_{1} /\left(b_{1}+b_{2}\right)$. The bet distribution for outcome 1 is

$$
p\left(b_{2}, b_{1} \mid 1\right)=\frac{N !}{b_{2} ! b_{1} !\left(N-b_{2}-b_{1}\right) !} \eta(2 \mid 1)^{b_{2}} \eta(1 \mid 1)^{b_{1}}[1-\eta(1 \mid 1)-\eta(2 \mid 1)]^{N-b_{2}-b_{1}},
$$

and likewise for $k=2$. Hence, $\left(1-\beta_{1}\right) / \beta_{1}=p\left(b_{2}, b_{1} \mid 2\right) / p\left(b_{2}, b_{1} \mid 1\right)=[\eta(1 \mid 2) / \eta(1 \mid 1)]^{b_{1}-b_{2}}$. If $\pi_{1}<1 / 2$, or $b_{2}>b_{1}$, the desired FLB inequality is $\left(1-\pi_{1}\right) / \pi_{1}<\left(1-\beta_{1}\right) / \beta_{1}$, i.e., $\left[\pi_{1} /\left[b_{1}\left(1-2 \pi_{1}\right)\right]\right] \log \left[\left(1-\pi_{1}\right) / \pi_{1}\right]<\log [\eta(1 \mid 1) / \eta(1 \mid 2)]$. This inequality holds for fixed $b_{2}$ and $b_{1}$ once the ratio $\eta(1 \mid 1) / \eta(1 \mid 2)$ is sufficiently large. From before, when $u \rightarrow \tau$ then $\hat{p}_{1} \rightarrow 1$. It follows from (12) that $\eta(1 \mid 1) / \eta(1 \mid 2)$ tends to infinity.

Proof of Proposition 8. Conditional on outcome $l$, the chance that $b_{k}=n$ is

$$
\operatorname{Pr}\left(b_{k}=n \mid l\right)=\left(\begin{array}{c}
N \\
n
\end{array}\right) \eta(k \mid l)^{n}[1-\eta(k \mid l)]^{N-n} .
$$


Conditional on $b_{k}=n$, the chance that $k$ wins is then

$$
\operatorname{Pr}\left(k \mid b_{k}=n\right)=\frac{q_{k} \eta(k \mid k)^{n}[1-\eta(k \mid k)]^{N-n}}{\sum_{j=1}^{K} q_{j} \eta(k \mid j)^{n}[1-\eta(k \mid j)]^{N-n}} .
$$

Given that the sampled outcome $z$ has market probability $\pi=n / N$, the relative chance that this outcome is $k$ is

$$
\operatorname{Pr}\left(k=z \mid b_{z}=n\right)=\frac{\operatorname{Pr}\left(b_{k}=n\right)}{\operatorname{Pr}\left(b_{z}=n\right)}=\frac{\sum_{j=1}^{K} q_{j} \eta(k \mid j)^{n}[1-\eta(k \mid j)]^{N-n}}{\sum_{m=1}^{K} \sum_{l=1}^{K} q_{l} \eta(m \mid l)^{n}[1-\eta(m \mid l)]^{N-n}} .
$$

Combining (14) and (15) we obtain the desired posterior probability that the sampled outcome with market probability $\pi=n / N$ is also the winning outcome:

$$
\sum_{k=1}^{K} \operatorname{Pr}\left(k \text { wins } \mid b_{k}=n\right) \operatorname{Pr}\left(k=z \mid b_{z}=n\right)=\frac{\sum_{k=1}^{K} q_{k} \eta(k \mid k)^{n}[1-\eta(k \mid k)]^{N-n}}{\sum_{m=1}^{K} \sum_{l=1}^{K} q_{l} \eta(m \mid l)^{n}[1-\eta(m \mid l)]^{N-n}} .
$$

We conclude that (7) is the expected return to a bet with market probability $\pi$. 


\section{Appendix B: Dirichlet Signal Structure}

This appendix introduces the Dirichlet signal structure, a tractable example that we use throughout the paper to illustrate our results. Suppose that the prior is symmetric, $q_{k}=1 / K$, and that signals unconditionally have the symmetric Dirichlet density

$$
g\left(s_{1}, \ldots, s_{K}\right)=\frac{\Gamma(K \theta)}{(\Gamma(\theta))^{K}} \prod_{k=1}^{K} s_{k}^{\theta-1}
$$

with full support on the simplex $\left\{s \in \mathbb{R}_{+}^{K} \mid \sum_{k=1}^{K} s_{k}=1\right\}$, where the parameter $\theta>0$ measures the inverse amount of private information and $\Gamma(\theta)=\int_{0}^{\infty} t^{\theta-1} e^{-t} d t=(\theta-1) \Gamma(\theta-1)$ denotes the Gamma function. The conditional distribution has density $K s_{k} g(s)$. Given symmetry in the prior, Bayes' rule implies posterior belief $p=s$, so that the the posterior belief $p$ follows the same Dirichlet distribution. The smaller is $\theta$, the more spread out is the distribution of $p$, verifying that $\theta$ is an inverse measure of information.

A particularly tractable special case of the Dirichlet example is obtained by setting $\theta=1$. This is the uniform signal example with conditional density $f(s \mid k)=c s_{k}$ where $c$ is a constant of integration. If the race is symmetric, $q_{k}=1 / K$, by Bayes' rule signal $s$ results in private belief $p=s$. Moreover, symmetric also ensures that the ex ante density of $p=s$ is uniform, $f(s)=\sum_{k=1}^{K} q_{k} f(s \mid k)=c / K$.

Proof of Lemma 1. When $K=2, \eta(1 \mid 1)$ is equal to the probability that $p_{1}>1 / 2$. Using integration by parts and $\frac{\Gamma(\theta+1) \Gamma(\theta)}{\Gamma(2 \theta+1)}=\int_{0}^{1} p_{1}^{\theta}\left(1-p_{1}\right)^{\theta-1} d p_{1}$, this probability satisfies

$$
\frac{\Gamma(\theta+1) \Gamma(\theta)}{\Gamma(2 \theta+1)} \eta(1 \mid 1)=\int_{1 / 2}^{1} p_{1}^{\theta}\left(1-p_{1}\right)^{\theta-1} d p_{1}=\frac{4^{-\theta}}{\theta}+\int_{1 / 2}^{1} p_{1}^{\theta-1}\left(1-p_{1}\right)^{\theta} d p_{1} .
$$

Substitution of $p_{2}=1-p_{1}$ gives

$$
\int_{1 / 2}^{1} p_{1}^{\theta-1}\left(1-p_{1}\right)^{\theta} d p_{1}=\int_{0}^{1 / 2} p_{2}^{\theta}\left(1-p_{2}\right)^{\theta-1} d p_{2}=\frac{\Gamma(\theta+1) \Gamma(\theta)}{\Gamma(2 \theta+1)}-\int_{1 / 2}^{1} p_{1}^{\theta}\left(1-p_{1}\right)^{\theta-1} d p_{1} .
$$

Collecting terms, we obtain

$$
2 \int_{1 / 2}^{1} p_{1}^{\theta}\left(1-p_{1}\right)^{\theta-1} d p_{1}=\frac{4^{-\theta}}{\theta}+\frac{\Gamma(\theta+1) \Gamma(\theta)}{\Gamma(2 \theta+1)}
$$

and hence (5). 
Proof of Lemma 2. By symmetry, it suffices to derive $\eta(1 \mid 1)$. Using $f(s \mid 1)=c s_{1}, s$ is Dirichlet distributed with parameters $(2,1, \ldots, 1)$. By Proposition 3, a bettor bets on outcome 1 when $s_{1}=p_{1}>p_{k}=s_{k}$ for all $k>1$.

Let $X_{1}, \ldots, X_{K}$ be independent $\chi^{2}$ distributed random variables, with degrees of freedom 4 for $X_{1}$ and 2 for $X_{2}, \ldots, X_{K}$. The distribution function for $X_{k}$ when $k>1$ is

$$
H(x)=1-e^{-x / 2} .
$$

Letting $r_{k}=X_{k} / \sum_{l=1}^{K} X_{l}$, Norman L. Johnson and Samuel Kotz (1972, Section 40.5) note that $r=\left(r_{1}, \ldots, r_{K}\right)$ follows the same Dirichlet distribution as $s$. The chance that $s_{1}>s_{k}$ for all $k>1$ is then equal to the chance that $X_{1}>X_{k}$ for all $k>1$. Given $X_{1}$, this chance is $H\left(X_{1}\right)^{K-1}$. Using the expression for the density of the $\chi^{2}$ distribution, we obtain

$$
\begin{aligned}
\eta(1 \mid 1) & =\frac{1}{4} \int_{0}^{\infty}\left(1-e^{-x / 2}\right)^{K-1} x e^{-x / 2} d x=\frac{1}{4} \int_{0}^{\infty}\left[\sum_{j=0}^{K-1}\left(\begin{array}{c}
K-1 \\
j
\end{array}\right)(-1)^{j} e^{-j x / 2}\right] x e^{-x / 2} d x \\
& =\frac{1}{4} \sum_{j=0}^{K-1}\left(\begin{array}{c}
K-1 \\
j
\end{array}\right)(-1)^{j} \int_{0}^{\infty} x e^{-(j+1) x / 2} d x=\sum_{j=0}^{K-1}\left(\begin{array}{c}
K-1 \\
j
\end{array}\right)(-1)^{j} \frac{1}{(j+1)^{2}},
\end{aligned}
$$

proving (6).

Proof of Lemma 3. Consider the case $k=1$, and let $X_{1}, \ldots, X_{K}$ and $r_{1}, \ldots, r_{K}$ be the same random variables as in the proof of Lemma 2. With full participation, outcome 1 is the best response when all $l \neq 1$ satisfy $X_{l} \leq X_{1} q_{1} W_{1} / q_{l} W_{l}$. Hence we obtain

$$
\eta(1 \mid 1)=\frac{1}{4} \int_{0}^{\infty} \prod_{l \neq 1}\left(1-e^{-\frac{x}{2} \frac{q_{k} W_{k}}{q_{l} W_{l}}}\right) x e^{-x / 2} d x
$$

Expanding the product, we have

$$
\prod_{l \neq 1}\left(1-e^{-\frac{x}{2} \frac{q_{k} W_{k}}{q_{l} W_{l}}}\right)=\sum_{j=0}^{K-1} \sum_{A \subseteq\{2, \ldots, K\},|A|=j}(-1)^{j} e^{-\frac{x}{2}\left(\sum_{m \in A} \frac{q_{k} W_{k}}{q_{m} W_{m}}\right)} .
$$

Equation (8) follows by integration. Recalling the different distribution of $X_{1}$ and $X_{l}$, a similar consideration gives

$$
\eta(l \mid 1)=\frac{1}{2} \int_{0}^{\infty}\left(1-\left(1+\frac{x}{2} \frac{q_{1} W_{1}}{q_{l} W_{l}}\right) e^{-\frac{x}{2} \frac{q_{1} W_{1}}{q_{l} W_{l}}}\right) \prod_{m \neq k, l}\left(1-e^{-\frac{x}{2} \frac{q_{k} W_{k}}{q_{m} W_{m}}}\right) e^{-\frac{x}{2}} d x .
$$

Again, expansion of the product and integration yields (9). 